\title{
Formation of Protein Carbonyls during Myocardial Reperfusion by Coronary Angioplasty
}

\author{
Jayakumari Narayani, ${ }^{1, *}$ Subramonialyer Krishna, ${ }^{1}$ and Balakrishnan K. Gopalakrishnan ${ }^{2}$ \\ ${ }^{1}$ Division of Biochemistry, Sree Chitra Tirunal Institute for Medical Sciences and Technology, Thiruvananthapuram \\ 695 011, Kerala, India \\ ${ }^{2}$ Department of Cardiology, Sree Chitra Tirunal Institute for Medical Sciences and Technology, Thiruvanan- \\ thapuram 695 011, Kerala, India
}

Received 9 March, 2002; Accepted 30 July, 2002

\begin{abstract}
Summary The quantitation of myocardial reperfusion injury in patients often creates problems due to lack of specific markers. Although malondialdehyde provides an index of oxidative damage, we have little information regarding effects on membrane structure and function. Therefore we investigated the effect of ischemia/reperfusion on the kinetics of protein modification along with lipid peroxidation during coronary angioplasty. Venous blood samples were collected before angioplasty and at various intervals after primary stage of balloon deflation from humans to quantitate the above parameters. A significant transient rise in the concentration of protein carbonyls in serum $(p<0.001)$ was observed soon after successful reperfusion which parallels with that of lipid peroxides. This increase of protein carbonyls is an actual reflection of the increased rate of amino acid modification in membrane proteins. These structural modifications can alter lipid-protein interactions resulting in disturbed membrane functions. This study suggests that protein modification and lipid peroxidation play significant role in the pathogenesis of myocardial reperfusion injury. Further this assessment of structural modification in proteins provides an important reliable technique to detect and quantitate the exact nature and extent of myocardial injury.
\end{abstract}

Key Words: protein carbonyls, lipid peroxides, reperfusion injury

\section{Introduction}

Progressive narrowing of the coronary artery in humans with atherosclerotic lesions is now treated with coronary angioplasty, which is a safe method of achieving early myocardial reperfusion. Reperfusion is necessary to reduce the size of the ischemic area and promote the recovery of energy metabolism of the myocardial area at risk. But it is inevitably associated with several harmful effects, which include both reversible events such as myocardial stunning and arrhythmogenesis and the more serious and irrevers-

\footnotetext{
*To whom correspondence should be addressed.
}

E-mail: jaya@sctimst.ker.nic.in ible lethal injury [1]. This hypothetical concept is termed reperfusion injury. Since the discovery that reintroduction of blood to ischemic tissue can initiate further damage of still viable cells, the mechanism of reperfusion injury has been a subject of intense investigation. It has been generally accepted that reperfusion of ischemic tissue is associated with the generation of reactive oxygen species (ROS) which presumably play an important role in the pathogenesis of ischemia/reperfusion injury [2-5]. ROS when generated in substantial quantity can injure even non-ischemic myocytes and endothelial cells. This mechanism of ischemia/reperfusion injury is commonly present during the release of coronary spasm, cardiac operations, successful thrombolytic therapy in acute myocardial infarction and coronary 
angioplasty and thus endangered with reperfusion injury through oxygen derived free radical mechanism [6]. However the exact nature of cellular damage produced by these radicals is not yet clear. It is therefore important to elucidate the underlying mechanisms that are responsible for cardiac dysfunction which may allow prevention of this common complications of severe myocardial ischemia/reperfusion.

Earlier we have conducted a study in human beings who underwent coronary artery bypass surgery to investigate the hypothesis of reperfusion injury and have demonstrated the occurrence of oxidative stress during the early phase of reperfusion using malondialdehyde (MDA) as an index of lipid damage [7]. Lipid peroxidation is considered a major mechanism of reactive oxygen species toxicity in reperfused heart. Free radical reactions are highly complex and we can expect metabolic and cellular disturbances to occur in various ways. Interventional studies have yielded inconsistent results and this led to the continued controversy over the role of ROS in myocardial reperfusion injury.

Although measurement of lipid peroxides provides an index of damage, they contribute little information about effects on membrane structure and function. There is growing evidence that ROS can directly attack the amino acids in proteins and can alter protein structure and function and proteolytic enzymes may minimize the effect of oxidative damage by preventing the accumulation of such altered proteins $[8,9]$. Indeed damage to proteins may often be more important than damage to lipids in oxidative stress situations in vivo [10]. Since ROS induced membrane damage is a highly complex biochemical process, we assume that enzymes and other structural proteins may also be damaged whenever toxic forms of oxygens are produced. It is therefore important to evaluate the possible modifications of proteins in order to understand the mechanism of the different biological effects of ROS. Therefore we proposed to investigate the effect of brief ischemia and reperfusion on the kinetic changes of protein modification in human beings during coronary angioplasty. Percutaneous transluminal coronary angioplasty is a widely used well accepted reperfusion treatment for the sclerotic lesions in patients which provide a unique model of controlled transient ischemia and reperfusion.

\section{Patients and Methods}

The study group consisted of 33 male coronary artery disease patients from the age group of 35-55 years who underwent percutaneous transluminal coronary angioplasty treatment for coronary artery disease in the Cardiology Department of our Institute. Angioplasty to the lesion related coronary artery was performed with standard technique and equipments, which involves conditions of transient repeated interruptions of coronary flow by the balloon occlusion sequences in patients. The strategy has been to attempt dilation of significant stenoses during a single procedure. After angioplasty all the patients recovered satisfactorily but only 26 patients benefited by this treatment, and have no acute reocclusion during the study period.

Venous blood samples were collected from each patient with informed consent before the start of angioplasty to provide the baseline values for this study. Subsequent venous blood samples were collected immediately after the first two consecutive balloon deflation sequences at 15,60 and $120 \mathrm{~s}$ and $2 \mathrm{~h}$ after completion of angioplasty procedure. Blood samples were centrifuged and sera were analyzed the same day for lipid peroxides and protein carbonyls.

Lipid peroxidation was assessed by measuring the thiobarbituric acid-reactive substances (TBARS) by the spectrophotometric method [11]. The absorbance of the resulting chromogen by the reaction of malondialdehyde formed from the breakdown of peroxidized polyunsaturated fatty acids with TBA was recorded at $535 \mathrm{~nm}$. For quantitation 1,1,3,3tetramethoxypropane was used as standard.

Protein carbonyls were quantitated by the method described by Levine et al. [12] using dinitrophenylhydrazine (DNPH). After precipitation of serum proteins with $20 \%$ trichloroacetic acid (TCA), the protein pellets were suspended in DNPH solution and incubated with shaking at $5 \mathrm{~min}$ interval at room temperature for $1 \mathrm{~h}$. After the DNPH reaction, proteins were reprecipitated with 20\% TCA and washed once with 10\% TCA and thrice with ethanol/ethyl acetate mixture $(1: 1)$ to remove lipids and excess DNPH. The precipitate was dissolved in $6 \mathrm{M}$ guanidine hydrochloride and the absorbance of the DNPH derivatives was recorded at $370 \mathrm{~nm}$ in UV-VIS spectrophotometer. The data were expressed as $\mu \mathrm{mol}$ of carbonyl group/liter serum by using a molar absorption coefficient of 21,000 $\mathrm{mol}^{-1}$. liter $\mathrm{cm}^{-1}$ for the DNPH derivatives. 
Data analysis was carried out with the use of paired ' $t$ ' test and analysis of variance.

\section{Results}

The data regarding the concentration of serum TBARS recorded at various time intervals from 16 patients after successful reperfusion are listed in Table 1.

There was a significant increase in the levels of serum TBARS soon after first stage of balloon deflation which reached a peak at $15 \mathrm{~s}(p<0.001)$ and afterwards the levels declined and almost normalized by about 2 min of reperfusion. The alteration in the concentration of TBARS at the second stage of balloon inflation followed a similar pattern to those observed after primary inflation stage, compared with pre-angioplasty values. In most of the cases maximum increase in the concentration of TBARS was noticed soon after the first stage of balloon inflation (first, $18 \%$ vs. second, $14 \%$ ).

The kinetic changes of the levels of serum protein carbonyls together with TBARS were available for a further group of patients $(n=10)$ after the primary stage of angioplasty and are shown in Fig. 1.

Serum protein carbonyls showed a significant transient increase soon after balloon deflation with a maximum increase $(39 \%, p<0.001)$ at $15 \mathrm{~s}$ of reperfusion compared with baseline value. The concomitant rise of TBARS was only $20 \%(p<0.001)$. After this initial peaking up both the parameters showed a decline in their concentration but were still significantly elevated than the pre-angioplasty value at 1 $\min (p<0.001)$ and reached a level just above the

Table 1. Changes in serum concentration of TBARS during the early phase of two consecutive stages of myocardial reperfusion by coronary angioplasty.

\begin{tabular}{lc}
\hline & TBARS as MDA $(\mu \mathrm{M})$ \\
\hline Baseline & $2.45 \pm 0.40$ \\
After first angioplasty & $2.89 \pm 0.50^{\mathrm{a}}$ \\
$15 \mathrm{~s}$ & $2.71 \pm 0.51^{\mathrm{b}}$ \\
$60 \mathrm{~s}$ & $2.54 \pm 0.42$ \\
$120 \mathrm{~s}$ & \\
After second angioplasty & $2.79 \pm 0.48^{\mathrm{a}}$ \\
$15 \mathrm{~s}$ & $2.73 \pm 0.50^{\mathrm{b}}$ \\
$60 \mathrm{~s}$ & $2.56 \pm 0.47$ \\
$120 \mathrm{~s}$ & \\
Post-angioplasty & $2.5 \pm 0.45$ \\
$2 \mathrm{~h}$ & as mean $\pm \mathrm{SD} \quad(n=16)$. \\
\hline The results are expressed ${ }^{\mathrm{a}} p<0.001$ vs. baseline; ${ }^{\mathrm{b}} p<0.01$ vs. baseline.
\end{tabular}

baseline by about $2 \mathrm{~min}$. Although there was a similarity between the pattern of alterations of these markers, after the immediate reperfusion period the peak values of protein carbonyls did not show any correlation $(\gamma=0.09)$ with that of TBARS.

Further in few cases $(n=7)$ the post-angioplasty levels of protein carbonyls (basal, $41.5 \pm 5.6 ; 15 \mathrm{~s}$, $42.1 \pm 4.5 ; 60 \mathrm{~s}, 40.9 \pm 4.9 ; 120 \mathrm{~s}, 42.3 \pm 4.7 ; 2 \mathrm{~h}, 42.5$ $\pm 5.1 \mu \mathrm{M}$ ) and TBARS (basal, $2.37 \pm 0.52 ; 15 \mathrm{~s}, 2.30$ $\pm 0.61 ; 60 \mathrm{~s}, 2.38 \pm 0.47 ; 120 \mathrm{~s}, 2.40 \pm 0.46 ; 2 \mathrm{~h}, 2.41$ $\pm 0.54 \mu \mathrm{M})$ did not reach any significant difference when compared with their corresponding basal values in whom the coronary angioplasty was unsuccessful.

\section{Discussion}

ROS may damage cells through a pathway dependent essentially on membrane damage, which has been the subject of extensive research over the past several years. While considerable efforts have been directed towards understanding the mechanism of ROS induced lipid peroxidation and its inhibition by antioxidants, little attention has been paid to the oxidative damage of proteins during situations of reperfusion injury. Among the various oxidative modification of proteins, carbonyl formation is believed to be an early marker of protein oxidation [10].

The present data showed that there was an abrupt transient rise in the serum concentration of protein carbonyls soon after successful reperfusion. The post-angioplasty changes of TBARS also parallels with that of protein carbonyls, but a maximum percentage rise was noticed in the formation of carbonyl groups at $\sim 15 \mathrm{~s}$ of reperfusion which indicates that these processes seem to occur by distinct mechanisms. Another important finding was that the postangioplasty concentration of these parameters remained unaltered in serum compared with their pre-angioplasty values in few patients, who were not benefited by this treatment, thus indicating that oxidative damage occurs only after successful recanalization by angioplasty. This transient rise of protein carbonyls and TBARS in serum was an actual reflection of the increased rate of amino acid oxidation in membrane proteins and lipid peroxidation respectively. Obviously these results were in agreement with the earlier reports $[13,14]$ demonstrating an increase in oxidation of proteins and lipids due to the generation of ROS in the isolated rat lung subjected to global ischemia and reperfusion. 


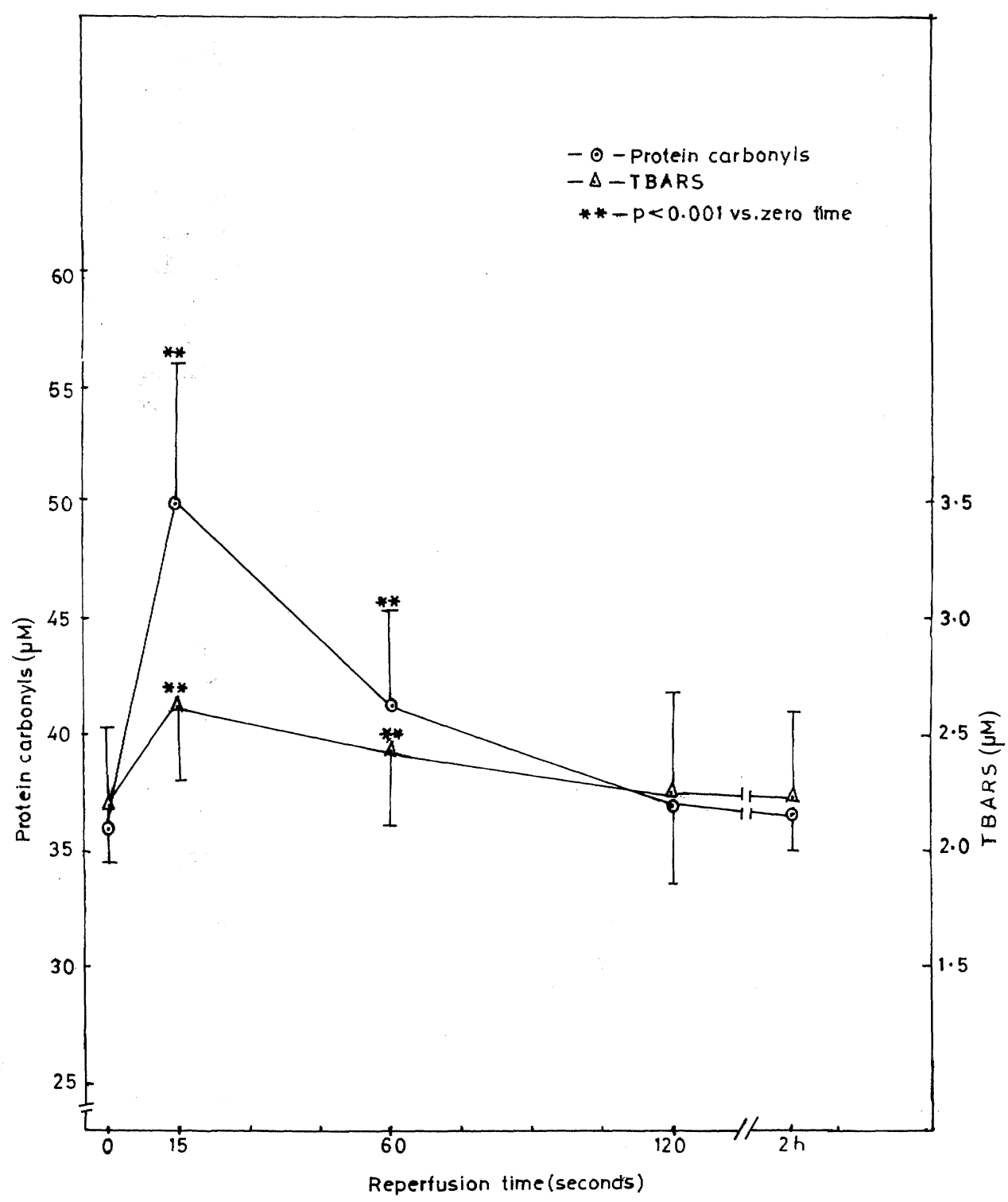

Fig. 1. Time course of the formation of protein carbonyls and TBARS in serum during early phase of myocardial reperfusion by coronary angioplasty. Mean \pm SD is presented $(n=10)$. $\odot$, protein carbonyls; $\triangle$, TBARS. ${ }^{* *} p<0.001$ vs. prereperfusion.

Electron spin resonance studies which involved the direct measurement of free radicals showed that oxygen free radicals remained at low concentration during the ischemic period but increased abruptly after reperfusion [15-17]. It is therefore likely that most of the protein carbonyls and TBARS might result from the complex macromolecular chain reactions induced by the ROS, produced at the time of reperfusion of the ischemic myocardium. Although the possibility for those being washed out by reperfusion from the pre-existing myocardial ischemic area and/or atherosclerotic lesion [18] cannot be ignored, this may partly contribute to the rise of these biochemical markers. The finding of a transient rise of TBARS during the early phase of reperfusion after angioplasty was in agreement with the previous reports $[19,20]$, which provide indirect evidence for the pathogenic role of ROS. However, Greech et al. [21] have found no alteration in the concentration of serum MDA after successful percutaneous transluminal coronary angioplasty, but reported elevated free radical activity as measured by percentage molar ratio of conjugated dienes. So far no other study has been reported identifying the 
kinetic changes in protein damage during situations of brief ischemia followed by reperfusion in human beings.

The precise mechanism of ROS generation and the cell damage during ischemia/reperfusion is not been completely understood. Available experimental evidence proposes that short reversible ischemia followed by reperfusion could result in increased production of ROS through several mechanisms including the activation of xanthine oxidase [22] and neutrophils [23]. The oxidative damage of proteins may often be more important than that of lipids in oxidative stress situations in vivo. In such situations of brief ischemia and reperfusion, the intensity of such damage may not be sufficient to cause myocardial cell death, but it is sufficient to produce dysfunction of key cellular organelles including sarcolemma, sacroplasmic reticulum, contractile proteins and extracellular collagen matrix. The membrane structure is essential for maintaining membrane integrity, permeability, ion-transport, membrane-bound receptors, enzyme systems, and organelle function. However in the present study we have not measured the association between these marker activity and the extent of cardiac functional alteration.

Coronary angioplasty provides a unique model of transient interruption of coronary flow by the balloon occlusion sequences (30-90 s) of lesion-related coronary artery in humans which enables one to study the myocardial metabolic changes under in vivo conditions of ischemia/reperfusion. The demonstration of oxidative injury indicates that amino acid modification of proteins is also taking place as a major mechanism of cell damage in addition to the well recognized mechanism of lipid peroxidation. When such structural modification occurs, this can alter lipid-protein interactions resulting in disturbed membrane function. These membrane changes together with calcium overload and mitochondrial abnormalities, subsequent to lipid peroxidation and protein modification, may be the underlying cause of reperfusion injury and associated cardiac dysfunction. In conclusion, this study suggests that ROS generated at the time of successful reperfusion by angioplasty elicit a variety of damaging reactions on proteins and lipids leading to reperfusion injury which is a potential threat to viable myocardium and may deny the patient the full benefit of reperfusion. Further the assessment of structural modification in proteins not only provides an important reliable biochemical technique for the quantitation of myocar- dial injury but also enables its use as a tool to evaluate the efficacy of different antioxidant therapies used to limit reperfusion injury.

\section{Acknowledgments}

The authors thank the Director, Sree Chitra Tirunal Institute for Medical Sciences and Technology for the facilities. They are also grateful to Ms. V. Ambikakumari and Mr. K.N. Vijayasenan for technical assistance.

\section{References}

[1] Kloner, A.R., Przyklenk, K., and Whittaker, P.: Deleterious effects of oxygen radicals in ischemia/reperfusion. Circulation, 80, 1115-1127, 1989.

[2] Thomson, J.A. and Hess, M.L.: The oxygen free radical system: a fundamental mechanism in the production of myocardial necrosis. Prog. Cardiovasc. Dis., 28, 449-462, 1986.

[3] Werns, S.W., Shea, M.J., and Lucchesi, B.R.: Free radicals in ischemic myocardical injury. J. Free. Radic. Biol. Med., 1, 103-110, 1985.

[4] McCord, J.M.: Oxygen derived free radicals in postischemic tissue injury. N. Engl. J. Med., 312, 159-163, 1985.

[5] Lucchesi, B.R.: Myocardial ischemia, reperfusion and free radical injury. Am. J. Cardiol., 65, 141-231, 1990.

[6] Hearse, P.J.: Free Radicals Oxidant Stress and Drug Actions, ed. by Rice-Evans, Richelicia Press, London, Vol. II, pp. 13-42, 1987.

[7] Jayakumari, N., Sunitha Kumari, K., Ambika Kumari, V., Nazar, Y., Mohan Singh, M.D., Balakrishnan, K.G., and Subramonialyer, K.: Enhanced lipid peroxidation in patients during coronary artery bypass grafting. Indian Heart J., 45, 489-491, 1993.

[8] Davies, K.J.A. and Goldberg, A.L.: Oxygen radical stimulate intracellular proteolysis and lipid peroxidation by independent mechanism in erythrocytes. $J$. Biol. Chem., 262, 8220-8227, 1989.

[9] Stadtman, E.R.: Oxidation of proteins by mixed function oxidation systems: Implication in protein turnover, ageing and neutrophil function. Trends Biol. Sci., 11, 11-12, 1986.

[10] Standtman, E.R. and Oliver, C.N.: Metal catalyzed oxidation of proteins. J. Biol. Chem., 266, 2005-2008, 1991.

[11] Burge, J.A. and Aust, S.D.: Thiobarbituric acid for lipid peroxides. Methods Enzymol., 52, 302-310, 1978.

[12] Levine, R.L., Garland, D., Oliver, C.N., Amics, A., Climent, I., Lenz, A.G., Ahn, B., Shaltiel, S., and Stadtman, S.: Determination of carbonyl content in oxidatively modified proteins. Methods Enzymol., 186, 464-478, 1990.

[13] Iyene, I.S., Dodia, C., and Fisher, A.B.: Role of oxygen in oxidation of lipid and protein during ischemial reperfusion in isolated perfused rat lung. Arch. Biochem. 
Biophys., 296, 183-189, 1992.

[14] Zhao, G., Iyere, I.S., and Fisher, A.B.: Role of iron in the ischemia-reperfusion oxidative injury of rat lungs. Am. J. Respir. Cell. Mol. Biol., 16, 293-299, 1992.

[15] Hess, M.L., Okaba, E., and Konton, H.A.: Proton and free oxygen radical interaction with the calcium transport system of cardiac sarcoplasmic reticulum. $J$. Mol. Cell. Cardiol., 13, 767-772, 1981.

[16] Bolli, R., Patel, B.S., Jeroudi, M.O., Lai, E.K., and Mclay, P.B.: Demonstration of free radical generation in 'stunned' myocardium of intact dog with the use of spin trap. J. Clin. Invest., 82, 476-485, 1988.

[17] Garlick, P.B., Davies, M.J., Hearse, D.J., and Slater, T.F.: Direct detection of free radicals in the reperfused rat heart using ESR spectroscopy. Circ. Res., 61, 756760, 1987.

[18] Ledwozyw, A., Michalak, J., Stepien, A., and Kadziolka, A.: The relationship between plasma triglyceride, cholesterol, total lipids and lipid peroxidation products during human atherosclerosis. Clin. Chim. Acta, 155, 275-284, 1986.

[19] Roberts, M.J.D., Young, I.S., Trouton, T.G., Trimble, E.R., Khan, M.M., Webb, S.W., Wilson, C.M.,
Patterson, G.C., and Adgey, A.A.J.: Transient release of lipid peroxides after coronary artery balloon angioplasty. Lancet, 336, 143-145, 1990.

[20] De Schrirder, I.K., Van de Kraag, A.M.M., Lamers, J.M.J., Koster, J.F., de Jong, J.W., and Serruys, P.W.: Myocardial MDA and uric acid release after short lasting coronary acclusion during coronary angioplasty, potential mechanism of free radical generation. $A m$. J. Cardiol., 68, 392-395, 1991.

[21] Greech, E.D., Bellamy, C.M., Jackson, M.J.J., Muirheed, R.A., Faragher, E.B., and Ramsdale, D.R.R.: Free radical activity after primary coronary angioplasty in acute myocardial infarction. Am. Heart J., 127, 1443-1447, 1994.

[22] Chambers, D.O.E., Parks, D.A., and Patterson, G.: Xanthine oxidase as a source of free radical damage in myocardial ischemia. J. Mol. Cell. Cardiol., 17, 145152, 1985.

[23] Reimer, K.A., Murry, C.E., and Richard, V.J.: The role of neutrophils and free radical in the ischemic reperfused heart. J. Mol. Cell. Cardiol., 21, 1225-1239, 1989. 